\title{
Chromosomal microarray analysis as a first-line test in pregnancies with a priori low risk for the detection of submicroscopic chromosomal abnormalities
}

\author{
Francesco Fiorentino $^{\star, 1}$, Stefania Napoletano ${ }^{1}$, Fiorina Caiazzo ${ }^{1}$, Mariateresa Sessa ${ }^{1}$, Sara Bono ${ }^{1}$, \\ Letizia Spizzichino ${ }^{1}$, Anthony Gordon ${ }^{2}$, Andrea Nuccitelli ${ }^{1}$, Giuseppe Rizzo ${ }^{1}$ and Marina Baldi ${ }^{1}$
}

In this study, we aimed to explore the utility of chromosomal microarray analysis (CMA) in groups of pregnancies with a priori low risk for detection of submicroscopic chromosome abnormalities, usually not considered an indication for testing, in order to assess whether CMA improves the detection rate of prenatal chromosomal aberrations. A total of 3000 prenatal samples were processed in parallel using both whole-genome CMA and conventional karyotyping. The indications for prenatal testing included: advanced maternal age, maternal serum screening test abnormality, abnormal ultrasound findings, known abnormal fetal karyotype, parental anxiety, family history of a genetic condition and cell culture failure. The use of CMA resulted in an increased detection rate regardless of the indication for analysis. This was evident in high risk groups (abnormal ultrasound findings and abnormal fetal karyotype), in which the percentage of detection was $5.8 \%(7 / 120)$, and also in low risk groups, such as advanced maternal age $(6 / 1118,0.5 \%)$, and parental anxiety $(11 / 1674,0.7 \%)$. A total of $24(0.8 \%)$ fetal conditions would have remained undiagnosed if only a standard karyotype had been performed. Importantly, 17 ( $0.6 \%$ ) of such findings would have otherwise been overlooked if CMA was offered only to high risk pregnancies.The results of this study suggest that more widespread CMA testing of fetuses would result in a higher detection of clinically relevant chromosome abnormalities, even in low risk pregnancies. Our findings provide substantial evidence for the introduction of CMA as a first-line diagnostic test for all pregnant women undergoing invasive prenatal testing, regardless of risk factors.

European Journal of Human Genetics (2013) 21, 725-730; doi:10.1038/ejhg.2012.253; published online 5 December 2012

Keywords: array comparative genomic hybridization; chromosomal microarray analysis; copy number variant; prenatal diagnosis; low risk pregnancies

\section{INTRODUCTION}

The widespread use of postnatal chromosomal microarray analysis (CMA) has shown submicroscopic rearrangements to represent a greater cause of genetic disorders than previously thought and they are currently associated with up to $15 \%$ of syndromic and nonsyndromic mental retardation. ${ }^{1-3}$

Much of the increased detection rate of CMA can be explained by its potential to detect chromosome abnormalities at significantly higher resolution, ${ }^{4-7}$ as compared to conventional cytogenetic methods, which may detect chromosomal anomalies to an average resolution of only $\sim 7-10 \mathrm{Mb}$ in size. CMA allows detection of microscopic and submicroscopic copy number variants (CNVs), as small as $50-100 \mathrm{~Kb}, \sim 100$ times smaller than the changes that can be identified by traditional karyotyping. ${ }^{7-10}$

CMA is now recognized as the appropriate first-line test, in place of conventional karyotyping, for the clinical evaluation of postnatal patients with developmental delay/intellectual disability, multiple congenital anomalies and/or autism spectrum disorders. ${ }^{3,4,6,8,11-14}$ This change from conventional karyotyping to CMA in postnatal cytogenetics has generated an increasing interest in determining whether CMA will offer any advantages for the detection of fetal chromosomal abnormalities in prenatal diagnostics.
Although several previously reported retrospective and prospective studies ${ }^{15-24}$ have demonstrated the effectiveness and usefulness of CMA in clinical prenatal diagnosis, only limited conclusions could be drawn due to the small size of the cohorts analyzed. These studies have all provided reassuringly consistent results in terms of analytical validity, clinical validity and clinical utility of the technique applied in the prenatal diagnosis setting, compared to traditional karyotyping. However, their limited data has necessitated undertaking of large-scale prospective clinical trials, with parallel processing of samples for both CMA and conventional cytogenetic analysis, before any firm conclusions can be drawn to recommend the technique as a firstline test in all prenatal samples. ${ }^{25}$

Recently, a large-scale prospective study ${ }^{26}$ was carried out by our center, in order to assess the feasibility and clinical advantages of offering CMA as a first-line test in clinical prenatal diagnosis. This study demonstrated the usefulness and benefits of prenatal diagnosis as performed by whole-genome CMA for direct analysis of amniocytes or CVS tissues, without culturing cells. Our study indicated that the CMA approach was robust, with no false positive findings when followed up with different methodologies, or false negative findings when samples were tested in parallel with conventional karyotyping. In addition to our study, other similar 
large cohort prospective trials have been published, all reporting results concordant with our findings. ${ }^{27-30}$

The above large-scale clinical trials, combined with previously reported small cohorts studies, have shown that the use of CMA in prenatal diagnosis produces a substantial improvement of $\sim 1-3 \%$ in detection rate of genetic aberrations compared with conventional karyotyping, without missing potentially pathogenic chromosomal abnormalities and with no appreciable increase in results of unclear clinical significance. These findings provided evidence for the feasibility of introducing CMA into routine prenatal diagnosis practice, indicating that it could be already acceptable to apply CMA as a first-line diagnostic test, at least concurrently with conventional karyotyping. ${ }^{26,29}$ However, at present, it is not yet clear what indication of prenatal diagnosis could benefit from CMA. In a recent meta-analysis of prenatal samples, ${ }^{31}$ the additive value of CMA was reported to be $5.2 \%$ when applied to pregnancies with abnormal ultrasound findings. However, the fact that only a minority of the submicroscopic abnormalities that are detected in postnatal patients with developmental delay/intellectual disability are accompanied by congenital abnormalities detectable by prenatal ultrasound, suggests the use of CMA in prenatal diagnostics not only for pregnancies with ultrasound abnormalities. ${ }^{32}$

In the absence of specific guidelines and large-scale studies for different categories of indications, it has been suggested that CMA should be offered as an adjunction tool to selected groups of high-risk pregnancies (eg abnormal ultrasound findings and a normal conventional karyotype), using the technique as a second-line test only, after a standard karyotype. ${ }^{25}$ Recently, the Canadian College of Medical Geneticists (CCMG) has discouraged the use of CMA in pregnancies at low risk for a structural chromosomal abnormality (eg advanced maternal age, positive maternal serum screen, previous pregnancy with a chromosomal abnormality or the presence of 'soft markers' on fetal ultrasound), motivating their position with the fact that CMA performed for the above categories of pregnancies would likely be associated with a low positive predictive value, since the vast majority of fetuses in these situations would be clinically unaffected. ${ }^{33}$

In this study, we aimed to explore the usefulness of CMA in groups of pregnancies with a priori low risk for the detection of submicroscopic chromosome abnormalities, usually not considered an indication for testing, in order to assess if CMA improves the prenatal detection rate of chromosomal aberrations.

\section{MATERIALS AND METHODS}

\section{Experimental design and clinical samples}

A large prospective trial was designed, involving analysis of 3000 prenatal samples and processed in parallel for both CMA and conventional cytogenetic analysis. CMA was offered as an option to couples considering an invasive prenatal genetic testing procedure, in addition to conventional karyotyping. Prenatal samples, inclusive of a subset of 1037 cases previously reported, ${ }^{26}$ were sent to a central karyotyping lab between October 2010 and March 2012 from 167 healthcare providers in Italy. Specimen types submitted included 2650 (88.3\%) amniotic fluid, $308(10.3 \%)$ chorionic villus sampling (CVS), 32 $(1.1 \%)$ cultured amniocytes and $10(0.3 \%)$ DNA extracted by other labs from uncultured amniocytes.

Patients underwent pre-test counseling as described elsewhere, ${ }^{34}$ during which the issues that are encountered with CMA were discussed. The couples who accepted evaluation by CMA signed an informed consent form containing a summary of the testing process, potential benefits and limitations of testing, and possible testing outcomes. A post-test genetic counseling session was provided in all cases when a genetic alteration was detected by any method. The study was approved by the Institutional Review Board of GENOMA laboratory.

\section{Indications for prenatal diagnosis}

The indications for invasive prenatal testing included: increased risk of fetal aneuploidy associated with advanced maternal age, that is 35 years or older at time of conception; parental anxiety, that is patients younger than 35 years with no specific indication for invasive prenatal diagnosis; abnormal results from maternal serum screening tests; abnormal ultrasound findings; a known abnormal fetal karyotype; family history of a genetic condition or chromosome abnormality; and cell culture failure.

\section{CMA and data interpretation}

Prenatal samples were processed according to the protocol reported elsewhere. $^{26}$ Briefly, DNA extracted from amniotic fluid, CVS and cultured amniocytes was first tested for gender determination of the fetus and maternal cell contamination. DNA samples were then processed by CMA using whole-genome bacterial artificial chromosome (BAC) microarraysCytoChip Focus Constitutional (BlueGnome, Cambridge, UK), according to the manufacturer's protocol. The genomic coverage of this microarray platform is up to $1 \mathrm{Mb}$ resolution across the genome and $\sim 100 \mathrm{~kb}$ resolution in 139 regions associated with constitutional disorders (more details available at http://www.cytochip.com/productfocus). Data analysis was performed by algorithm fixed settings in BlueFuse Multi Software (BlueGnome, Cambridge, UK). The minimum number of microarray probes used for calling a copy number change was at least 3 contiguous BAC clones. Detected CNVs were compared to known CNVs in publicly available databases (eg Database of Genomic Variants - DGV; Decipher etc.) and in our own database of results and classified as clinically significant, likely benign or as variants of uncertain clinical significance (VOUS), as reported elsewhere. ${ }^{14,26}$ Briefly, results were reported as 'normal' (no clinically significant CNVs detected, with or without benign CNVs), 'abnormal' (with clinically significant copy-number changes containing dosage-sensitive or disease-causing genes or occurring within a region of the genome known to be involved in syndromes associated with chromosome abnormalities), or with a 'VOUS', defined as an alteration of unclear clinical relevance that has not been previously identified in a laboratory's patient population, not been reported in the medical literature, not been found in publicly available databases or does not contain any known disease-causing genes. The size cut-off for was $0.5 \mathrm{Mb}$ for the backbone and $100 \mathrm{~Kb}$ for disease-focused regions The microarray findings were confirmed as previously described. ${ }^{26}$

\section{RESULTS}

A total of 3000 prenatal samples were processed in parallel using both CMA and G-banding for conventional karyotyping.

The indications for prenatal testing included: advanced maternal age $(n=1118 ; 37.3 \%)$, abnormal results from maternal serum screening tests $(n=29 ; 1.0 \%)$, abnormal ultrasound findings $(n=95 ; 3.2 \%)$, abnormal fetal karyotype $(n=25 ; 0.8 \%)$, family history of a genetic condition $(n=25 ; 0.8 \%)$, parental anxiety $(n=1675 ; 55.8 \%)$ and cell culture failure $(n=33 ; 1.1 \%)$. Successful results were obtained by CMA in 3000/3000 (100\%) of cases, $98.9 \%$ of which was achieved using only uncultured material. The majority of prenatal samples $(2905 / 3000 ; 96.8 \%)$ had normal results, with no copy number changes or only benign CNVs identified. Clinically significant chromosome alterations were identified in $95(3.2 \%)$ samples, $71(74.7 \%)$ of which were also detected by conventional karyotyping.

Numerical chromosome anomalies were detected in $54(1.8 \%)$ fetuses, including 35 with trisomy 21, 9 with trisomy 18, 3 with trisomy 13, 2 with 45,X, 1 with 47,XXY, 2 with 47,XXX and 2 with 47,XYY. Microscopic segmental chromosomal deletions or duplications were diagnosed in $6(0.2 \%)$ fetuses. CMA was also able to detect chromosomal mosaicism in $11(0.4 \%)$ samples, with the lowest abnormal chromosome representation being at the $6 \%$ level.

In 24 cases $(0.8 \%$ of the entire cohort of samples and $25.3 \%$ of the clinically relevant findings), CMA identified pathogenic $\mathrm{CNV}$ s that 
would have remained overlooked if only a conventional karyotype had been performed, 18 of which mapped to regions associated with well-established syndromes. Notably, $6.3 \%$ of cases with a normal karyotype and abnormal ultrasound findings had either a microdeletion or duplication of potential or known clinical significance, as did $0.5 \%$ of those sampled for maternal age or $0.7 \%$ for parental anxiety.

The details of the chromosomal abnormalities detected are listed in Supplementary Material and are presented below in relation to the indications for prenatal diagnosis.

\section{Abnormal ultrasound findings}

CMA detected a pathological chromosomal imbalance in 26/95 $(27.4 \%)$ fetuses with abnormal ultrasound findings: 8 with trisomy 21,4 with trisomy 18,1 with trisomy 13,5 with mosaic aneuploidies and 2 with microscopic segmental chromosomal deletion or duplication. Submicroscopic chromosomal aberrations were detected in 6 $(6.3 \%)$ fetuses, 2 of which presented with congenital heart disease (tetralogy of Fallot), 2 with abnormal nuchal translucency and 1 with cystic hygroma. In one fetus with single umbilical artery, a $3.4 \mathrm{Mb}$ inherited deletion at $17 \mathrm{p} 12$ was detected, consistent with hereditary neuropathy with liability to pressure palsies, a chromosomal abnormality not directly related to the ultrasound findings.

\section{Advanced maternal age}

Among the 1118 cases sampled for maternal age indication, CMA detected chromosome anomalies in $34(3.0 \%)$ fetuses: 14 with trisomy 21, 4 with trisomy 18, 1 with trisomy 13, 5 with sex chromosomes aneuploidies and 4 with mosaic aneuploidies. Pathogenic submicroscopic CNVs were detected in $6(0.5 \%)$ fetuses, 5 (cases 6-10, 12) of which mapped to regions associated with wellestablished syndromes, as described in the Online Mendelian Inheritance in Man (OMIM) database; one (case 11) affecting a male fetus, consisted of a $3.3 \mathrm{Mb}$ inherited (maternal) deletion at Xp11.3p11.23 and was classified as clinically significant $\mathrm{CNV}$, because it was involving a chromosomal region containing clinically relevant genes.

\section{Parental anxiety}

Among the 1675 fetuses without a specific prenatal risk, CMA detected numerical chromosome anomalies in 28 (1.7\%) fetuses: 11 with trisomy 21,1 with trisomy 18,1 with trisomy 13,2 with sex chromosomes aneuploidies and 2 with segmental duplications, one of which was mosaic. Pathogenic submicroscopic CNVs were detected in $11(0.7 \%)$ fetuses, nine (cases 13-18, 20, 21 and 23) of which mapped to known syndromes. Case 22, was shown to have a de novo unbalanced translocation between chromosomes 7 and 9, resulting in a $7.3 \mathrm{Mb}$ duplication at $7 \mathrm{q} 36.1 \mathrm{q} 36.3$ and a $9.6 \mathrm{Mb}$ deletion at $9 \mathrm{p} 24.3 \mathrm{p} 23$. Case 19 was shown to have a de novo $7.5 \mathrm{Mb}$ duplication at $19 \mathrm{q} 13.41 \mathrm{q} 13.43$. The above de novo aberrations, involving large chromosomal regions and containing clinically relevant genes, were classified as clinically significant CNVs.

\section{Known abnormal fetal karyotype}

A total of 25 samples were investigated by CMA because an abnormal fetal karyotype was detected. Eleven samples contained an apparently balanced chromosomal translocation or inversion; in 5 samples de novo small supernumerary marker chromosomes (sSMC) were detected, 4 samples were investigated because of a suspected segmental structural chromosomal abnormality and 5 samples were presented because a chromosomal mosaicism was detected following CVS karyotyping. CMA confirmed the suspected segmental chromosomal abnormality in 2 samples and in one sample it was helpful for correctly scoring of the aberration (a duplication 15q24.1-qter instead of a $5 \mathrm{q}$ duplication). In one sample, CMA allowed the identification of the origin of the sSMC. In one sample referred because of an apparently de novo balanced chromosomal rearrangement [46,XY,der(6)inv(6)(p12;q14)t(6;8)p21.1;q23)], CMA detected a $5.2 \mathrm{Mb}$ deletion at breakpoint 6q14.3 (Case 24).

\section{Abnormal maternal serum screening test}

CMA detected a numerical chromosome abnormality in 3/29 (10.3\%) fetuses with abnormal results of maternal serum screening tests: 2 were trisomy 21 and one was a $20 \%$ mosaic for trisomy 18 . No submicroscopic chromosomal aberrations were detected for this indication.

Family history of a genetic condition or chromosomal abnormality No genetic imbalances were detected in any of the 25 cases sampled for this indication.

\section{Results of unclear clinical significance}

A single CNV of unclear significance, out of 3000 samples tested, was detected in an amniotic fluid sample referred with indication of parental anxiety. It consisted in a $0.34 \mathrm{Mb}$ de novo deletion at 7q11.23 [arr 7q11.23 (71209746×2, 72 049391-72418522 × , $72747995 \times 2)$ ], proximal to the Williams-Beuren syndrome (WBS) critical region. This deletion included the FKBP6 gene, which has been shown to play a role in homologous chromosome pairing in meiosis and male fertility in mouse models. ${ }^{35,36}$ The involvement of the typical WBS loci was excluded by fluorescent in situ hybridization (FISH), using a commercially available probe mapping to the Elastin gene, as commonly used for confirmation of the deletion associated with WBS. After post-test counseling, the patient decided to continue with pregnancy.

\section{DISCUSSION}

Whether CMA should be used in prenatal diagnosis as a first-line test in routine practice has been widely debated. ${ }^{37-39}$ At present, numerous independent prospective studies, ${ }^{17-19,22-24,26-30}$ involving the use of various strategies and validated with several different array platforms, have demonstrated the effectiveness and usefulness of CMA in prenatal diagnosis compared to conventional karyotyping.

In our large-scale prospective study, a figure of $0.8 \%$ represented the average improvement in the detection rate of CMA compared with traditional karyotyping, concordant with those reported by other similar studies. ${ }^{27-30}$ The combined data from published prospective studies represent now a cohort of over 13000 prenatal samples $^{7-19,22-24,26-30}$ (Table 1). Taken together, their findings clearly indicate that the use of CMA in prenatal diagnosis produces a substantial improvement in the detection rate of pathogenic chromosome abnormalities compared with conventional karyotyping. Reports to date suggest that CMA is a more sensitive diagnostic test and adds incremental value to conventional karyotyping, with the potential to replace karyotyping in the initial cytogenetic evaluation of the fetus.

The results of this study provide particular evidence on the improved diagnostic ability of CMA to detect clinically relevant abnormalities and the utility of bringing CMA into routine prenatal diagnosis practice as a primary diagnostic tool with a number of different indications. Even more clearly, it indicates that offering only CMA as second-line test just to high-risk pregnancies, may substantially limit the diagnostic potential of prenatal diagnosis, since a significant proportion of $\mathrm{CNV}$ s that can cause a serious disability will not be detected by traditional karyotyping. The most relevant clinical 
Table 1 Comparison of CMA detection rates in prospective prenatal studies

\begin{tabular}{|c|c|c|c|}
\hline Reference & $\begin{array}{c}\text { No. of prenatal samples } \\
\text { analyzed }\end{array}$ & $\begin{array}{c}\text { No. of clinically } \\
\text { significant submicroscopic aberrations (\%) }\end{array}$ & $\begin{array}{l}\text { No. of CNVs of unclea } \\
\text { significance (\%) }\end{array}$ \\
\hline Sahoo et all ${ }^{17}$ & 98 & $0(0.0)$ & $2(2.0)$ \\
\hline Shaffer et all ${ }^{18}$ & 151 & $2(1.3)$ & $1(0.7)$ \\
\hline Kleeman et $a^{21}$ & 50 & $1(2.0)$ & $0(0.0)$ \\
\hline Coppinger et $a^{R 2}$ & 244 & $5(2.0)$ & $1(0.4)$ \\
\hline Van de Veyver et aR3 & 300 & $2(0.7)$ & $3(1.0)$ \\
\hline Maya et $a R^{4}$ & 269 & $3(1.1)$ & $0(0.0)$ \\
\hline Park et aR7 & 4.033 & $11(0.3)$ & NR \\
\hline Armengol et $a{ }^{28}$ & 906 & $14(1.5)$ & $6(0.7)^{\mathrm{a}}$ \\
\hline Lee et $a^{29}$ & 3.171 & $34(1.1)$ & $5(0.2)$ \\
\hline Breman et $a^{\beta 0}$ & 1.115 & $25(2.2)$ & $18(1.6)$ \\
\hline Current study & 3.000 & $24(0.8)$ & $1(0.03)$ \\
\hline Total & 13.337 & $121(0.9)$ & $37(0.3)$ \\
\hline
\end{tabular}

aonly not inherited CNVs are taken into consideration.

point in regard to this argument relates to the 24 submicroscopic chromosomal abnormalities $(0.8 \%$ of the entire cohort of samples and $25.3 \%$ of the clinically relevant findings) that would have remained undiagnosed if only a conventional karyotype had been performed. Yet more interestingly, $17(0.6 \%)$ of these karyotypically cryptic findings would have been otherwise overlooked following the diagnostic strategy of offering CMA only to high-risk pregnancies. Similar results have also been reported by other independent largescale prospective studies (Table 1). ${ }^{26-30}$

A point to note was how CMA was able to robustly detect copy numbers changes in all cases, such as case 2 (in which the G-banded karyotyping failed to detect the chromosomal abnormality) and case 3 (in which a cell culture failure occurred). For these cases, a good quality traditional karyotyping at the 400-band level should have been able to reliably detect the aberrations, but it failed to do so. It is well known the resolution of banding techniques is generally influenced by the quality of the chromosome preparation, thus it is not always possible to achieve the desired resolution in routine prenatal banding analysis. This is all the more true when performing karyotyping from CVS samples, as it was for case 2, where cytogenetic preparations are often technically challenging. Cases 2 and -3 represent clear examples of inherent limitations of the use of traditional karyotyping, but not in CMA.

The evidence presented here makes CMA use in a routine prenatal diagnosis practice a proposition that needs to be considered. In fact, the chromosomal abnormalities that can be exclusively detected by CMA are not always age-related and considering the frequency of these disorders, it could be argued that this technique could be offered to all women undergoing invasive prenatal diagnosis, irrespective of the indication for testing. ${ }^{40}$ Ogilvie et al ${ }^{41}$ estimated that, using CMA, there is a 1:300-1:600 $(0.33-0.16 \%)$ chance of finding a CNV that will cause a serious disability, which can not be otherwise detected by traditional karyotyping.

The results of the present study are clearly in favor of the above hypothesis, although it shows that Ogilvie et $a l^{41}$ estimations to be underestimations, as our data shows the use of CMA results in an increased detection rate regardless of the indication for analysis. This becomes especially evident when examining the data from high-risk groups (abnormal ultrasound findings + abnormal fetal karyotype), in which the detection rate is elevated to $5.8 \%$ (7/120), but also in groups with a priori low risk for the detection of submicroscopic chromosome abnormalities, such as advanced maternal age (6/1118,
$0.5 \%)$ and parental anxiety $(11 / 1675,0.7 \%)$ groups, with an average increase in detection rate of $0.6 \%$ for low risk pregnancies (Table 2).

In the recent paper from Lee et $a l,{ }^{29}$ a figure of about $0.5 \%$ represented the average improvement in detection rate in low-risk pregnancies over conventional prenatal cytogenetics; Armengol et al, ${ }^{28}$ instead, reported a figure of $1.1 \%$ for advanced maternal age group and $1.7 \%$ for parental anxiety group. The combined data from prospective studies represent a cohort of over 8000 prenatal samples, referred for different categories of indication and indicates an average probability of detecting pathogenic submicroscopic CNVs in prenatal samples from low-risk pregnancies of around $0.6 \%$ (see Supplementary Material). Furthermore, a large prospective multicenter trial involving analysis of over 4000 samples, sponsored by the National Institute Of Child Health and Human Development (NICHD), investigating the role of CMA as a primary prenatal diagnostic tool, has been recently completed and its anticipated results $^{42}$ report data concordant with our study. This level of increased detection rate may suggest that, instead of restricting the use of CMA only to fetuses with ultrasound anomalies, this technology should be available to all pregnant women undergoing invasive prenatal testing, regardless of risk factors.

There is understandable concern regarding the use of CMA on prenatal samples, mainly related to the potential detection by CMA, of mild or unpredictable phenotypes and/or VOUS. ${ }^{37,43}$ However, it is well described that the differences in the proportion of VOUS detected is mainly related to the array platform used and its resolution. In our study, we have carefully selected a genome wide BAC-array platform specifically developed for prenatal applications, with a balance of increased resolution $(\sim 100 \mathrm{~kb})$ in locations of known constitutional disorders as well as less coverage in polymorphic CNVs and in backbone (up to $1 \mathrm{Mb}$ resolution), compared with high resolution oligonucleotide or single-nucleotide polymorphism (SNP) microarray platforms. While higher resolution, high resolution microarrays offer the theoretical opportunity to find additional submicroscopic abnormalities compared to BAC arrays and are the default array choice for postnatal cytogenetics. However, the detection rate for high- and low-risk pregnancies found in the present study using a BAC array platform, compares well with other large scale prospective studies $28,29,31,42$ in which high resolution microarrays have been used.

There are clear issues with the use of these platforms for prenatal diagnostics. The high resolution detection potential of oligonucleotide 
No. Samples with a pathogenic CNV (\%)

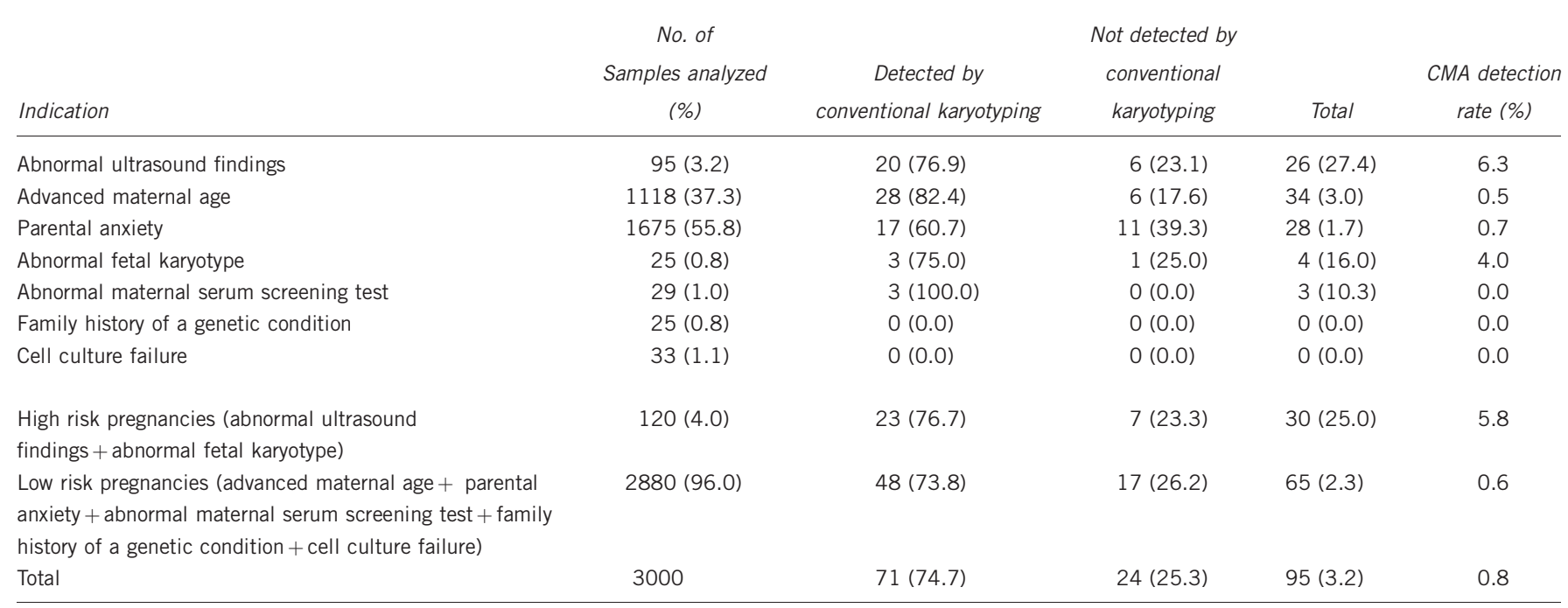

or SNP microarrays is offset in prenatal cases with more complex aberration calling issues due to the higher noise introduced when analyzing the poorer quality/quantity prenatal samples on these platforms. High resolution platforms also often require DNA extraction from cultured cells, which adds to diagnostic delays/costs and was not the case here for BAC arrays. Most importantly, the BAC array design strategy used here resulted in a substantially lower detection of variants of unclear significance (only a single VOUS occurrence out of 3000 samples analyzed), the central prenatal microarray diagnostic issue, than reported by other studies using high resolution microarrays (Table 1 ).

In a recent systematic review, ${ }^{31}$ VOUS are reported to occur in $\sim 1 \%$ of prenatal samples. Combining the above data with previously reported prospective studies, the average probability of detecting such findings in prenatal samples can be estimated approximately $0.3 \%$ (Table 1). It is also well known that the frequency of detection of VOUS increases if parental samples are not available, since the inheritance status of the VOUS cannot be ascertained. However if parental samples can be submitted with the prenatal samples, parental testing can be performed as soon as a fetal chromosomal aberration is identified, thereby alleviating additional timescale related anxiety for the patients and substantially reducing the number of uncertain calls.

The point to debate is surely whether we are able to or willing to deal with such level of VOUS. We believe that this potential uncertainty is not dramatically different from what is observed with routine prenatal cytogenetic karyotype analysis. In fact, results of unclear significance are not unfamiliar in traditional prenatal diagnosis because unclear diagnostic results or findings with unclear clinical consequences are occasionally encountered even with conventional karyotyping. Thus, VOUS identified by prenatal CMA might be approached in a similar manner and managed by providing the patients a thorough pre-test and post-test counselling. ${ }^{34}$ This will allow patients to achieve the maximum autonomy and right to information from CMA in order to make informed choices about their pregnancies. ${ }^{44}$

Although detection of VOUS cannot be completely excluded with CMA, the growing clinical experience with genome-wide arrays, the increasing availability, sophistication and size of CNV databases (in house or public) for both phenotypically normal and affected individuals, together with parental analysis, allows classification and interpretation for the majority of the alterations discovered, thus substantially reducing the number of VOUS and making CMA increasingly applicable to clinical prenatal diagnosis practice.

We believe that the improved prenatal diagnosis provided by CMA compared with the traditional karyotype outweighs the potential uncertainties for families. We also think that some patients would prefer to have maximum information and autonomy. ${ }^{45}$

In conclusion, the results of this study suggest that more widespread CMA testing of fetuses would result in a higher detection of clinically relevant chromosome abnormalities, even in low risk pregnancies. Our findings, combined with data from previous studies, provide substantial evidence for the introduction of CMA as a firstline diagnostic test for all pregnant women undergoing invasive prenatal testing, regardless of risk factors. We believe that the diagnostic accuracy of CMA may offer a much more fine-grained tool for prenatal diagnosis than other currently available technologies, allowing patients to achieve an improved prenatal diagnosis, with a lower risk for the newborn to be affected by a chromosomal abnormality. Of course, its introduction into clinical practice should proceed with caution, being offered only by experienced laboratories and after a proper internal validation of the technique showing robust, reliable, reproducible and possibly externally validated results.

\section{CONFLICT OF INTEREST}

The authors declare no conflict of interest.

\section{ACKNOWLEDGEMENTS}

We would like to thank Anna Iorillo, Elena Dangelosante and Nello Vitale for their valuable technical assistance. We are also very grateful to all healthcare providers participating in the study for their important contribution in patient referral and clinical management.

1 Vissers LE, de Vries BB, Osoegawa $\mathrm{K}$ et al: Array-based comparative genomic hybridization for the genomewide detection of submicroscopic chromosomal abnormalities. Am J Hum Genet 2003; 73: 1261-1270. 
2 de Vries BB, Pfundt $\mathrm{R}$, Leisink $\mathrm{M}$ et al: Diagnostic genome profiling in menta retardation. Am J Hum Genet 2005; 77: 606-616.

3 Stankiewicz P, Beaudet AL: Use of array CGH in the evaluation of dysmorphology, malformations, developmental delay, and idiopathic mental retardation. Curr Opin Genet Dev 2007; 17: 182-192.

4 Cheung SW, Shaw CA, Yu W et al: Development and validation of a CGH microarray for clinical cytogenetic diagnosis. Genet Med 2005; 7: 422-432.

5 Roa BB, Pulliam J, Eng CM, Cheung SW: Evolution of prenatal genetics: from point mutation testing to chromosomal microarray analysis. Expert Rev Mol Diagn 2005; 5 883-892.

6 Lu X, Shaw CA, Patel A et al: Clinical implementation of chromosomal microarray analysis: summary of 2513 postnatal cases. PLOS ONE 2007; 2: e327.

7 Shaffer LG, Bejjani BA, Torchia B, Kirkpatrick S, Coppinger J, Ballif BC: The identification of microdeletion syndromes and other chromosome abnormalities: Cytogenetic methods of the past, new technologies for the future. Am J Med Genet C Semin Med Genet 2007; 145C: 335-345.

8 Bejjani BA, Saleki R, Ballif BC et al: Use of targeted array-based CGH for the clinical diagnosis of chromosomal imbalance: is less more? Am J Med Genet A 2005; 134 259-267.

9 Emanuel BS, Saitta SC: From microscopes to microarrays: dissecting recurrent chromosomal rearrangements. Nat Rev Genet 2007; 8: 869-883.

10 Shaffer LG, Theisen A, Bejjani BA et al: The discovery of microdeletion syndromes in the post-genomic era: review of the methodology and characterization of a new 1q41q42 microdeletion syndrome. Genet Med 2007; 9: 607-616.

11 Shaffer LG, Kashork CD, Saleki R et al: Targeted genomic microarray analysis for identification of chromosome abnormalities in 1500 consecutive clinical cases. $J$ Pediatr 2006; 149: 98-102.

12 Rauch A, Hoyer J, Guth S et al: Diagnostic yield of various genetic approaches in patients with unexplained developmental delay or mental retardation. Am J Med Genet 2006; 140: 2063-2074.

13 Hochstenbach R, van Binsbergen E, Engelen J et al: Array analysis and karyotyping: workflow consequences based on a retrospective study of 36,325 patients with idiopathic developmental delay in the Netherlands. Eur J Med Genet 2009; 52: 161-169.

14 Miller DT, Adam MP, Aradhya S et al: Consensus statement: Chromosomal microarray is a first-tier clinical diagnostic test for individuals with developmental disabilities or congenital anomalies. Am J Hum Genet 2010; 86: 749-764.

15 Le Caignec C, Boceno M, Saugier-Veber P et al: Detection of genomic imbalances by array based comparative genomic hybridisation in fetuses with multiple malformations. J Med Genet 2005; 42: 121-128.

16 Rickman L, Fiegler H, Shaw-Smith $\mathrm{C}$ et al: Prenatal detection of unbalanced chromosomal rearrangements by array CGH. J Med Genet 2006; 43: 353-361.

17 Sahoo T, Cheung SW, Ward P et al: Prenatal diagnosis of chromosomal abnormalities using array-based comparative genomic hybridization. Genet Med 2006; 8: 719-727.

18 Shaffer LG, Coppinger J, Alliman S et al: Comparison of microarray-based detection rates for cytogenetic abnormalities in prenatal and neonatal specimens. Prenat Diagn 2008; 28: 789-795.

19 Bi W, Breman AM, Venable SF et al: Rapid prenatal diagnosis using uncultured amniocytes and oligonucleotide array CGH. Prenat Diagn 2008; 28: 943-949.

20 Vialard F, Molina GD, Leroy B et al: Array comparative genomic hybridization in prenatal diagnosis: another experience. Fetal Diagn Ther 2009; 25: 277-284

21 Kleeman L, Bianchi DW, Shaffer LG et al: Use of array comparative genomic hybridization for prenatal diagnosis of fetuses with sonographic anomalies and normal metaphase karyotype. Prenat Diagn 2009; 29: 1213-1217.

22 Coppinger J, Alliman S, Lamb AN et al: Whole-genome microarray analysis in prenatal specimens identifies clinically significant chromosome alterations without increase in results of unclear significance compared to targeted microarray. Prenat Diagn 2009, 29: $1156-1166$.

23 Van den Veyver IB, Patel A, Shaw CA et al: Clinical use of array comparative genomic hybridization (aCGH) for prenatal diagnosis in 300 cases. Prenat Diagn 2009; 29: 29-39.
24 Maya I, Davidov B, Gershovitz L et al: Diagnostic utility of array-based comparative genomic hybridization (aCGH) in a prenatal setting. Prenat Diagn 2010; 30 1131-1137.

25 ACOG committee. Array comparative genomic hybridisation in prenatal diagnosis. Obstet Gynecol 2009; 114: 1161-1163.

26 Fiorentino F, Caiazzo F, Napolitano S et al: Introducing array comparative genomic hybridization into routine prenatal diagnosis practice: a prospective study on over 1000 consecutive clinical cases. Prenat Diagn 2011; 31: 1270-1282.

27 Park SJ, Jung EH, Ryu RS et al: Clinical implementation of whole-genome array CGH as a first-tier test in 5080 pre and postnatal cases. Mol Cytogenet 2011; 9: 4-12.

28 Armengol L, Nevado J, Serra-Juhé C et al: Clinical utility of chromosomal microarray analysis in invasive prenatal diagnosis. Hum Genet 2012; 131: 513-523.

29 Lee CN, Lin SY, Lin CH et al: Clinical utility of array comparative genomic hybridization for prenatal diagnosis: A cohort study of 3171 pregnancies. BJOG 2012; 119: 614-625.

30 Breman A, Pursley AN, Hixson P et al: Prenatal chromosomal microarray analysis in a diagnostic laboratory; experience with $>1000$ cases and review of the literature. Prenat Diagn 2012; 32: 351-361.

31 Hillman SC, Pretlove S, Coomarasamy A et al: Additional information from array comparative genomic hybridization technology over conventional karyotyping in prenatal diagnosis: a systematic review and meta-analysis. Ultrasound Obstet Gynecol $2011 ; 37:$ 6-14.

32 Strassberg M, Fruhman G, Van den Veyver IB: Copy-number changes in prenatal diagnosis. Expert Rev Mol Diagn 2011; 11: 579-592.

33 Duncan A, Langlois SSOGC Genetics Committee; CCMG Prenatal Diagnosis Committee. Use of array genomic hybridization technology in prenatal diagnosis in Canada. $J$ Obstet Gynaecol Can 2011; 33: 1256-1259.

34 Darilek S, Ward P, Pursley A et al: Pre- and postnatal genetic testing by arraycomparative genomic hybridization: genetic counseling perspectives. Genet Med 2008; 10: 13-18.

35 Crackower MA, Kolas NK, Noguchi J et al: Essential role of Fkbp6 in male fertility and homologous chromosome pairing in meiosis. Science 2003; 300: 1291-1295.

36 Metcalfe K, Simeonov E, Beckett W, Donnai D, Tassabehji M: Autosomal dominant inheritance of Williams-Beuren syndrome in a father and son with haploinsufficiency for FKBP6. Clin Dysmorphol 2005; 14: 61-65.

37 Friedman JM: High-resolution array genomic hybridization in prenatal diagnosis Prenat Diagn 2009; 29: 20-28.

38 Pergament E: Controversies and challenges of array comparative genomic hybridization in prenatal genetic diagnosis. Genet Med 2007; 9: 596-599.

39 Bui TH, Vetro A, Zuffardi O, Shaffer LG: Current controversies in prenatal diagnosis 3: is conventional chromosome analysis necessary in the post-array CGH era? Prenat Diagn 2011; 31: 235-243.

40 Vetro A, Bouman K, Hastings R et al: The introduction of arrays in prenatal diagnosis: a special challenge. Hum Mutat 2012; 33: 923-929.

41 Ogilvie CM, Yaron Y, Beaudet AL: Current controversies in prenatal diagnosis 3: For prenatal diagnosis, should we offer less or more than metaphase karyotyping? Prenat Diagn 2009; 29: 11-14

42 Wapner RJPrenatal Microarray NICHD Study Group. A multicenter, prospective, masked comparison of chromosomal microarray with standard karyotyping for routine and high risk prenatal diagnosis. Am J Obstet Gynecol 2012; 9: 206:S2.

43 Shuster E: Microarray genetic screening: a prenatal roadblock for life? Lancet 2007, 369: 526-529.

44 McGillivray G, Rosenfeld JA, McKinlay Gardner RJ, Gillam LH: Genetic counselling and ethical issues with chromosome microarray analysis in prenatal testing. Prenat Diagn 2012; 32: 389-395.

45 Srebniak M, Boter M, Oudesluijs G et al: Application of SNP array for rapid prenatal diagnosis: implementation, genetic counselling and diagnostic flow. Eur J Hum Genet 2011; 19: 1230-1237.

Supplementary Information accompanies the paper on European Journal of Human Genetics website (http://www.nature.com/ejhg) 